\title{
Correction to: Will I have to move because of climate change? Perceived likelihood of weather- or climate-related relocation among the US public
}

\author{
Byungdoo Kim ${ }^{1} \cdot$ David L. Kay ${ }^{2} \cdot$ Jonathon P. Schuldt ${ }^{1}$
}

Published online: 12 April 2021

(C) Springer Nature B.V. 2021

\section{Correction to: Climatic Change (2021) 165:9 \\ https://doi.org/10.1007/s10584-021-03026-y}

The article was published without the Abstract. This has been corrected.

\begin{abstract}
As threats from climate change increase across the world, climate-induced migration is receiving more attention, particularly as it affects people in the Global South. However, despite increasing extreme weather events in nations including the USA, limited research has examined public perceptions of the personal likelihood of migration among members of wealthy industrialized countries. We asked a national probability sample of 1000 U.S. adults about the likelihood that they would have to relocate in the next 10 years because of weather or climate-related issues. We found that a majority of respondents $(57.0 \%)$ reported that these would have a "moderate" or greater influence on their decision to move in the coming decade. Logistic regression analysis with demographic (race, gender, socioeconomic status, and political party affiliation) and geographic indicators (regional division and coastal proximity) revealed that Democrats and people with higher education were more likely to indicate that weather- or climate-related issues would factor into their decision to relocate. Implications for climate change migration and public opinion research are discussed.
\end{abstract}

The online version of the original article can be found at https://doi.org/10.1007/s10584-021-03026-y

Byungdoo Kim

bk483@cornell.edu

1 Department of Communication, Cornell University, Ithaca, NY 14853, USA

2 Department of Global Development, Cornell University, Ithaca, NY, USA 